\title{
Microwave Interstellar Medium Emission Observed by theWilkinson Microwave Anisotropy Probe
}

\section{Citation}

Finkbeiner, Douglas P. 2004. "Microwave Interstellar Medium Emission Observed by theWilkinson Microwave Anisotropy Probe." The Astrophysical Journal 614 (1) (October 10): 186193. doi:10.1086/423482.

\section{Published Version}

doi:10.1086/423482

\section{Permanent link}

http://nrs.harvard.edu/urn-3:HUL.InstRepos:33462891

\section{Terms of Use}

This article was downloaded from Harvard University's DASH repository, and is made available under the terms and conditions applicable to Other Posted Material, as set forth at http:// nrs.harvard.edu/urn-3:HUL.InstRepos:dash.current.terms-of-use\#LAA

\section{Share Your Story}

The Harvard community has made this article openly available.

Please share how this access benefits you. Submit a story.

Accessibility 


\title{
MICROWAVE INTERSTELLAR MEDIUM EMISSION OBSERVED BY THE WILKINSON MICROWAVE ANISOTROPY PROBE
}

\author{
Douglas P. Finkbeiner ${ }^{1}$ \\ Department of Astrophysics, Princeton University, Peyton Hall, Princeton, NJ 08544 \\ Received 2003 July 1; accepted 2003 December 5
}

\begin{abstract}
We investigate the nature of the diffuse Galactic emission in the Wilkinson Microwave Anisotropy Probe $(W M A P)$ temperature anisotropy data. Substantial dust-correlated emission is observed at all WMAP frequencies, far exceeding the expected thermal dust emission in the lowest frequency channels $(23,33$, and $41 \mathrm{GHz})$. The WMAP team interprets this emission as dust-correlated synchrotron radiation, attributing the correlation to the natural association of relativistic electrons produced by supernovae $(\mathrm{SNe})$ with massive star formation in dusty clouds, and deriving an upper limit of 5\% on the contribution of Draine \& Lazarian spinning dust to the $\mathrm{K}$ band $(23 \mathrm{GHz})$. We pursue an alternative interpretation that much, perhaps most, of the dust-correlated emission at these frequencies is indeed spinning dust, and explore the spectral dependence on environment by considering a few specific objects as well as the full-sky average. Models similar to Draine \& Lazarian spinning dust provide a good fit to the full-sky data. The full-sky fit also requires a significant component with a flat spectrum uncorrelated with $\mathrm{H} \alpha$, possibly hot $\left(\sim 10^{6} \mathrm{~K}\right)$ gas within $30^{\circ}$ of the Galactic center.
\end{abstract}

Subject headings: cosmic microwave background — dust, extinction - ISM: clouds radiation mechanisms: thermal — radio continuum: ISM

Online material: color figure

\section{INTRODUCTION}

The first year of data from the Wilkinson Microwave Anisotropy Probe (WMAP) has sparked a revolution in the study of cosmology and the early universe (Bennett et al. 2003; Hinshaw et al. 2003; Spergel et al. 2003). In addition, it has provided full-sky maps of the interstellar medium at 23$94 \mathrm{GHz}$ at a sensitivity of $\sim 200 \mu \mathrm{K}$ per $7^{\prime}$ pixel at resolutions much higher than previous full-sky observations. The quality of the data has rekindled the debate about the origin of "anomalous" dust-correlated microwave emission (Kogut et al. 1996; de Oliveira-Costa et al. 1997, 1998, 1999, 2002, 2004; Finkbeiner et al. 2002, 2004; Banday et al. 2003; Lagache 2003) dubbed "foreground X" by de Oliveira-Costa et al. (2002).

The WMAP team relied on a maximum entropy method (MEM) analysis to determine that spinning dust ${ }^{2}$ with the Draine \& Lazarian (1998) cold neutral medium (CNM) spectrum accounts for less than $5 \%$ of the emission in any WMAP waveband. We show that for different models of spinning dust, these constraints do not apply. Indeed, in most of the Galaxy, spinning dust emission (or some other mechanism, such as magnetic dipole emission; Draine \& Lazarian 1999) explain the data just as well.

We emphasize that the WMAP team's primary objective was to prevent Galactic foreground emission from contaminating their cosmological results based on the cosmic microwave background (CMB) anisotropy. They have done a superb job of modeling the foregrounds, regardless of whether the physical

\footnotetext{
1 Hubble Fellow, Henry Norris Russell Fellow.

2 The idea of grain rotational emission was first discussed by Erickson (1957). More recently, after the discovery of the population of ultra small grains, Ferrara \& Dettmar (1994) noted that the rotational emission from such grains may be observable, but their treatment incorrectly assumed Brownian thermal rotation of grains.
}

interpretation of the subtracted temperature anisotropy signal is correct. The WMAP polarization data also provide important cosmological constraints as well as additional information about foregrounds. For example, if the Bennett et al. (2003) hard synchrotron component can be shown to be $\sim 30 \%$ polarized, then spinning dust would become an unlikely explanation (see Lazarian \& Draine 2000). On the other hand, if it is unpolarized, the synchrotron would be ruled out. Something between the two extremes is likely, and this will help us determine the relative contributions of synchrotron and spinning dust. As the polarization data are not yet public, discussion of polarization will be deferred to a later paper.

\section{ANALYSIS}

A disadvantage of the MEM analysis used by the WMAP team is that it can only find the components it is searching for. Significant spectral variation is expected not only in the synchrotron spectrum, but also in any spinning dust that might be present. The WMAP strategy, using data at the WMAP frequencies and priors based on the Haslam et al. (1982) $408 \mathrm{MHz}$ map, the Finkbeiner (2003) H $\alpha$ map, and the Finkbeiner et al. (1999; hereafter FDS99) dust prediction, produces a "synchrotron" template highly correlated with thermal dust emission (see Fig. 4 in Bennett et al. 2003). The explanation for this is that the most energetic electrons are found nearest dusty starforming regions, so there is a natural correlation between dust and synchrotron emission. This is certainly plausible, but it is not the only possible explanation for the strong correlation with dust.

Instead, we start by asserting that there are three components whose spectral characteristics are well enough understood to use external data sets, subtract these off in fits with very little freedom, and then approach the remaining emission with an open mind. These three components are free-free, soft 
synchrotron, and thermal dust. Here we differentiate between "soft" synchrotron (traced by the Haslam et al. [1982] map at $408 \mathrm{MHz}$ ) and "hard" synchrotron as described by Bennett et al. (2003). The existence of the soft synchrotron is not in dispute.

\subsection{Free-Free}

Although warm ionized gas is well traced by $\mathrm{H} \alpha$ emission in three recent surveys (VTSS, Dennison et al. 1998; SHASSA, Gaustad et al. 2001; WHAM, Haffner et al. 2003) as presented by Finkbeiner (2003) and corrected for dust using the Schlegel et al. (1998; hereafter SFD98) dust map, one must worry about several systematics. Bennett et al. (2003) point out several sources of uncertainty, paraphrased as follows: (1) $\mathrm{H} \alpha$ light scatters off dust, while microwave free-free scattering is negligible; (2) $\mathrm{H} \alpha$ calibration is uncertain at the $10 \%$ level; (3) for the $1 / 4$ of the sky with no WHAM coverage, there is significant uncertainty $(\sim 1 \mathrm{R})$ separating geocoronal $\mathrm{H} \alpha$ from Galactic interstellar medium (ISM) emission; (4) the assumed case B Balmer atomic rates may not be universally valid; (5) the ratio of $\mathrm{H} \alpha$ to free-free microwave emission depends on $T_{e}$, which may vary from object to object (e.g., Heiles et al. 2000); (6) there is uncertainty in the ionization state of $\mathrm{He}$; (7) ionized gas with bulk velocity outside of the WHAM velocity coverage is not included in the Finkbeiner map; and (8) the extinction correction assumes that the ionized gas and dust are coextensive along the line of sight. Of these sources of error, (5) and (8) likely dominate, while (2), (3), and (6) are well characterized, and (1) and (4) are probably small.

In spite of these difficulties, we can still make use of an $\mathrm{H} \alpha$ template. The first step is to limit the $\mathrm{H} \alpha$ extinction predicted by the SFD98 dust map to be $A<2$ mag. For dust with optical depth $\tau=A /(1.086 \mathrm{mag})$ uniformly mixed with $\mathrm{H} \alpha$ emitting gas the observed intensity is

$$
I_{\nu, \mathrm{obs}}=\frac{I_{\nu}}{\tau} \int_{0}^{\tau} d \tau^{\prime} e^{-\tau^{\prime}}=\frac{I_{\nu}}{\tau}\left(1-e^{-\tau}\right) .
$$

In the limit of large $\tau$, the intensity is simply reduced by a factor of $\tau$. For $A=2$ mag the uniform mixing case results in a reduction factor of 2.2, between the extremes of dust in front of (factor of 6.8) and dust behind (factor of unity) the gas. Although uniform mixing appears to be a reasonable approximation in general, we bear in mind that factor of $\sim 2$ errors in the free-free correction are likely in some parts of the sky.

A systematic variation in $T_{e}$ is also expected, with lower temperatures toward the Galactic center, where higher metallicity allows for higher cooling efficiency. Lacking an electron temperature map, we assume $T_{e}=5500 \mathrm{~K}$ everywhere, and use the free-free "haze" (§ 3.3) to absorb the errors in this. Using the conversion formula of Valls-Gabaud (1998), an emission measure $(\mathrm{EM})$ of $1 \mathrm{~cm}^{-6} \mathrm{pc}$ corresponds to $0.391 T_{4}^{-1.017} \times$ $10^{-0.029 / T_{4}}$ Rayleigh $\left(T_{4}=T_{e} / 10^{4} \mathrm{~K}\right)$, which equals $0.636 \mathrm{R}$ for $T=5500 \mathrm{~K}$.

Once an EM is derived for a given pixel on the sky, the spectrum is constrained by physics (Spitzer 1978), so only one free parameter $\left(T_{e}\right)$ is needed for the whole sky. This is valid between the plasma frequency cutoff and the optically thick limit at low frequencies. According to catalogs (Kurtz et al. 1994) of optically thick ultracompact H II regions, the filling factor of such regions is so small that at WMAP frequencies a negligible fraction of the free-free is expected to be optically thick, and certainly within our rather conservative mask there can be no significant ultracompact $\mathrm{H}$ II regions.

\subsection{Soft Synchrotron}

Synchrotron emission from relativistic electrons with energy distribution $N(E) \sim E^{-\gamma}$ produces a synchrotron flux density spectral index $\alpha=-(\gamma-1) / 2$. In temperature units the index is $\beta=-(\gamma+3) / 2$.

The electron energy distribution is expected to vary spatially in the Galaxy, with a harder spectrum near the sources of energetic electrons, e.g., supernova remnants, and a softer spectrum elsewhere. Bennett et al. (2003) argue that the natural correlation of supernova remnants with dusty star-forming clouds should cause a strong correlation between hard synchrotron emission (at $\sim 30 \mathrm{GHz}$ ) and dust, with the correlation lessening at lower frequencies, so that at $408 \mathrm{MHz}$, the Haslam et al. (1982) "soft" synchrotron map shows very little correlation to dust, other than the usual concentration of emission in the Galactic plane. This is certainly a cogent argument that synchrotron emission should become more dust-correlated at higher frequencies, but provides no evidence that such radiation dominates other mechanisms, such as spinning dust.

There is little doubt, however, that soft synchrotron (traced by the $408 \mathrm{MHz}$ survey) is present and important at least at $\mathrm{K}$ band $(23 \mathrm{GHz})$, so we include it in the removal of "known components." A brightness temperature index of $\beta=-3.05$ removes the most obvious K-band synchrotron features at high latitude, so we assume this index is constant throughout the high-latitude sky.

\subsection{Thermal Dust}

Another component whose morphology and (approximate) spectral characteristics are understood is thermal (vibrational) dust emission from grains large enough to be in equilibrium with the interstellar radiation field. Emission from this dust peaks at $\sim 140 \mu \mathrm{m}$ and deviates strongly from a thermal blackbody spectrum. A Rayleigh-Jeans emissivity function of $\nu^{2}$ has often been assumed in the literature (e.g., Draine \& Lee 1984; SFD98), but when dust temperature variation is accounted for, the COBE FIRAS data (Fixsen et al. 1996) are better fitted by a steeper power law emissivity $(\beta=2.6)$ near the peak and $\beta=1.7$ at lower frequencies, with a break at about $500 \mathrm{GHz}$ (Finkbeiner et al. 1999; FDS99). This fit ties the IRAS and DIRBE data to FIRAS via a fit with only four global parameters describing the two emissivity laws, and the requirement that the emission is dominated by grains in equilibrium with the interstellar radiation field. Predictions at $6^{\prime}$ resolution based on this fit are available on the Web. ${ }^{3}$

Because the spectral slope used to extrapolate the FDS99 thermal dust spectrum through the WMAP bands is relevant to this work, we briefly review its justification. The FDS99 twocomponent model yields a substantially better fit (reduced $\chi^{2}=$ 1.85 compared to 31 for a $\nu^{2}$ model) even when the spatial and spectral covariance of the FIRAS data are included (FIRAS Explanatory Supplement, Brodd et al. 1997). The FIRAS data cannot differentiate between a single component with nonpower-law emissivity and two independent power-law components, but the physical reality of the two separate components is not implausible. Amorphous silicates with a wide range of emissivity indices $\beta \sim 1.2-2.7$ have been observed in the lab (Agladze et al. 1996), including amorphous $\mathrm{MgO} \cdot 2 \mathrm{SiO}_{2}$, which has a very high microwave emissivity to optical absorption ratio, leading to rather different mean temperatures ( 9 and $16 \mathrm{~K}$ ) for the two components. These lab emissivities were measured

\footnotetext{
${ }^{3}$ See http://skymaps.info.
} 
at $\sim 300 \mathrm{GHz}$ and $20 \mathrm{~K}$ and may become steeper at lower frequencies. However, this interpretation of the spectral break is hardly unique; if the dominant emitter has such a break in its emissivity function at $500 \mathrm{GHz}$, then a single component could explain the data just as well. Another explanation that has been advanced is very cold dust grains spatially mixed with the warm dust (Reach et al. 1995), although a physical mechanism for keeping the grains so cold is not provided. Such a model would predict a steeper slope at lower frequencies also.

Regardless of interpretation, the Finkbeiner et al. (1999) model has been very successful in the submillimeter and microwave, although small but interesting deviations from the model have been observed by BOOMERANG (150, 240, and $410 \mathrm{GHz}$; Masi et al. 2001). At lower frequencies, however, there is a surprise.

Comparing FDS99 predictions to $C O B E$ DMR, Finkbeiner et al. (1999) found that $C O B E 90 \mathrm{GHz}$ was slightly higher, but at 53 and $31 \mathrm{GHz}$ the emission per dust column is a factor of 2.2 and 31 higher than expected, respectively. These results are similar to the earlier Kogut et al. (1996) results derived without an explicit dust temperature correction. Because of this it was expected that the FIRAS-based predictions would agree well with WMAP $94 \mathrm{GHz}$, but be significantly contaminated by some other dust-correlated emission mechanism at lower frequencies. This appears to be true.

Even though we can expect the vibrational thermal dust brightness temperature to go as $T \sim \nu^{\beta}$ with $\beta=2$ through the $W M A P$ channels, there may be other emissivity enhancements, such as magnetic dipole emission. These are treated as a separate component, so that temperature and other environmental dependence can be searched for as well.

The WMAP team (Bennett et al. 2003) has determined that the spectral slope from 94 to $61 \mathrm{GHz}$ is $\beta=2.2$, significantly steeper than the $\beta=1.7$ extrapolated from the Finkbeiner et al. (1999) fit. However we find in this work that $\beta=1.7$ is consistent with the data, and lacking any good theoretical reason to depart from the FDS99 parameterization, we continue to use this index in the following.

\subsection{Spinning Dust Template}

For a spinning dust template we use an empirically determined power of dust color temperature (derived from the DIRBE $100 \mu \mathrm{m} / 240 \mu \mathrm{m}$ ratio) times column density. This template reflects the observed $T_{\text {dust }}$ dependence of the anomalous emission, but assumes constant spectral dependence across the sky. We use $T_{\text {dust }}^{2}$ times the FDS99 prediction at $94 \mathrm{GHz}$, which is proportional to dust column times $T_{\text {dust }}^{3}$ because the Rayleigh-Jeans dust prediction already contains one power of temperature. This modulation by $T_{\text {dust }}^{3}$ varies the dust template by a factor of $\sim 2$ peak to peak for the SFD98 temperature range $(16-20 \mathrm{~K})$. There is no obvious theoretical reason for this temperature dependence other than the vague notion that hotter dust tends to be in a more exciting radiation field, or perhaps has a size distribution with more small grains, either one of which might enhance spinning dust emission. We will fit this average spectrum to the (nearly) full sky and then examine a few regions of interest to see deviations from it.

\subsection{CMB Anisotropy}

The CMB dipole is subtracted by the WMAP team and used to calibrate the anisotropy pattern to less than $1 \%$ uncertainty. The WMAP sensitivity is high enough that CMB anisotropy is clearly visible in all bands and (for this work) represents a significant nuisance background signal. Because the cosmological signal is not correlated with the foreground morphology for any physical reason, the cross-correlation between the foreground templates and the CMB (due only to chance alignment of features) is minimal. Nevertheless, we use the superposition of the five bands suggested by Bennett et al. (2003) to estimate the CMB anisotropy, and subtract it from each band. The superposition used is

$$
\begin{aligned}
\Delta T_{\mathrm{CMB}}= & 0.109 T_{\mathrm{K}}-0.684 T_{\mathrm{Ka}}-0.096 T_{\mathrm{Q}}+1.921 T_{\mathrm{V}} \\
& -0.250 T_{\mathrm{W}},
\end{aligned}
$$

where $T_{\mathrm{K}}, T_{\mathrm{Ka}}, T_{\mathrm{Q}}, T_{\mathrm{V}}$, and $T_{\mathrm{W}}$ refer to the thermodynamic $\triangle T$ in the five $W M A P$ bands, respectively. This works quite well except where bright Galactic point sources near the ecliptic plane show the beam asymmetries. ${ }^{4}$ This superposition only imperfectly cancels the foregrounds, of course, allowing them to contaminate the $\mathrm{CMB}$ estimate and leak back into our foreground analysis. We find that any such contamination is negligible, as it would appear as a foreground with the spectrum of the CMB, and no such residual is found in our analysis.

\subsection{Mask}

We mask out the 208 sources listed in the WMAP source catalog, and also the LMC, SMC, M31, Orion-Barnard's Loop, and NGC 5090 (a total of $2.7 \%$ of the sky). In addition, areas where the $\mathrm{H} \alpha$ extinction given by SFD98 is greater than 2 mag, i.e., $A(\mathrm{H} \alpha) \equiv 2.65 E(B-V)>2$, are masked (8.9\%). The mask is smoothed to $1^{\circ}$ FWHM to match the smoothed data, excluding a total of $11.4 \%$ of the sky from the analysis.

\subsection{The Fit}

The fit minimizes $\chi^{2}$ in an eight-dimensional parameter space over unmasked pixels. First all data and templates are smoothed to $1^{\circ}$ resolution using spherical harmonic convolution on the $N=512$ HEALPix $^{5}$ sphere. Noise is derived from $\sigma_{0}$ values and $N_{\text {obs }}$, the number of observations in each pixel (Jarosik et al. 2003). Initial guesses are determined by extensive experimentation with the data, then passed to the simpleminded but fairly robust amoeba routine from Numerical Recipes (Press et al. 1992) as implemented in IDL. ${ }^{6}$ Although the fit is a simultaneous regression on all parameters, it is instructive to "peel away" the foregrounds one at a time and show each WMAP band at each step.

Figure 1 is a six column by seven row grid of images, each a Cartesian projection of half the sky $\left(-90^{\circ}<l<90^{\circ}\right.$; $\left.-90^{\circ}<b<90^{\circ}\right)$. The first five columns are the WMAP bands in frequency order $(\mathrm{K}, \mathrm{Ka}, \mathrm{Q}, \mathrm{V}, \mathrm{W})=(23,33,41,61$, $94 \mathrm{GHz}$ ). The sixth column is the template being subtracted from the current row to yield the images on the row below. The first column is stretched so that $-1 \mathrm{mK}$ is black and $1 \mathrm{mK}$ is white $\left( \pm 15 \mathrm{Jy} \mathrm{sr}^{-1}\right)$. Columns (2)-(4) are stretched to the same intensity limits (in $\mathrm{Jy} \mathrm{sr}^{-1}$ ) as column (1), and column (5) matches the stretch of column (4) in thermodynamic

\footnotetext{
${ }^{4}$ It is often said that the inherently asymmetric WMAP beams are symmetrized by scanning each point on the sky from many different approach angles. This is true near the ecliptic poles, but at low ecliptic latitude the beam asymmetry is obvious in the K- and Ka-band maps.

5 See http://www.eso.org/science/healpix. Smoothing is done with spherical harmonic convolution on the HEALPix sphere, using smoothing code implemented in IDL by D. Finkbeiner.

${ }^{6}$ IDL is a product of Research Systems, Inc., http://rsinc.com.
} 


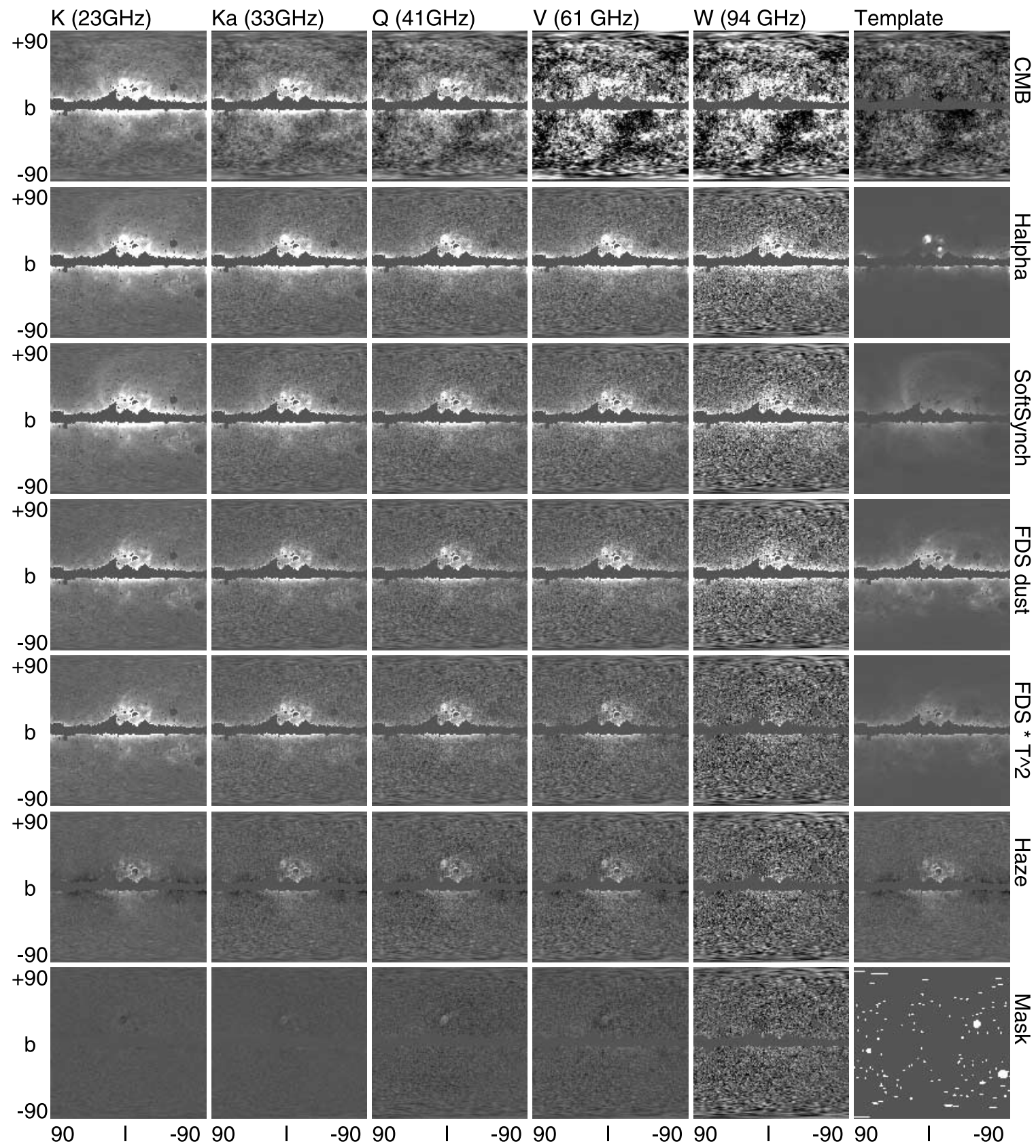

FIG. 1.-WMAP foreground grid; see detailed discussion in $\S 2.7$.

temperature units (to avoid saturation of the gray scale). Therefore, the CMB appears to become stronger from columns (1) through (4), but is the same brightness in column (5) as column (4).

Row (1).- The sky signal (after CMB dipole removal) for the 5 WMAP bands, with the plane and point sources masked. Column (6) is the CMB map described in equation (2).

Row (2).-CMB-subtracted sky; note the $\mathrm{H}$ in region around $\zeta$ Oph, above the plane and just left of center. This feature is prominent in the $\mathrm{H} \alpha$ based free-free template, shown at right. The free-free spectrum is constrained by physics, so only one parameter is fit for an overall normalization.

Row (3).-Sky minus CMB and $\mathrm{H} \alpha$; note the "Loop I" synchrotron feature in the north, visible in the Haslam-based soft synchrotron template at right. A single power law is fit from the Haslam et al. (1982) survey frequency (408 MHz) to WMAP. The spectrum is likely concave downward over such a large range in frequency, but WMAP $23 \mathrm{GHz}$ dominates the fit, and is the only frequency for which this component is important anyway.

Row (4).- Sky minus CMB, $\mathrm{H} \alpha$, and soft synchrotron. Dustcorrelated emission is evident, as traced by the Finkbeiner et al. (1999) template at right. This template is subtracted with amplitudes determined by the FDS99 fit to FIRAS, not by crosscorrelation with the WMAP data. Note that the subtraction at $94 \mathrm{GHz}$ appears to be nearly perfect.

Row (5).--Sky minus $\mathrm{CMB}, \mathrm{H} \alpha$, soft synchrotron, and FDS99 dust. There is still significant dust-correlated emission present at lower frequencies. The FDS99 dust emission template times $T_{\text {dust }}^{2}$ (SFD98 dust temperature squared, based on DIRBE 100 and $240 \mu \mathrm{m}$ emission ratio) appears to be a good template for the remaining component, tentatively interpreted as spinning dust, and is shown at right. A cross-correlation coefficient is determined independently for each WMAP band. 
TABLE 1

$\chi^{2}$ Results, Single Band

\begin{tabular}{|c|c|c|c|c|c|c|c|}
\hline $\begin{array}{l}\text { Map } \\
\text { (1) }\end{array}$ & $\begin{array}{c}b \text {-Cut } \\
\text { (deg) } \\
\text { (2) }\end{array}$ & $\begin{array}{c}\text { K Band } \\
(23 \mathrm{GHz}) \\
(3)\end{array}$ & $\begin{array}{c}\text { Ka Band } \\
(33 \mathrm{GHz}) \\
\text { (4) }\end{array}$ & $\begin{array}{c}\text { Q Band } \\
(41 \mathrm{GHz}) \\
(5)\end{array}$ & $\begin{array}{c}\text { V Band } \\
(61 \mathrm{GHz}) \\
(6)\end{array}$ & $\begin{array}{c}\text { W Band } \\
\text { (94 GHz) } \\
(7)\end{array}$ & $\begin{array}{c}\text { Mean } \\
(8)\end{array}$ \\
\hline W & all & 391.8645 & 139.7677 & 93.6773 & 43.6718 & 31.5367 & 140.1036 \\
\hline W & $<30$ & 583.3032 & 206.6596 & 133.5638 & 54.6387 & 38.4774 & 203.3285 \\
\hline W-C & all & 122.9073 & 17.7629 & 8.7606 & 7.0739 & 2.2803 & 31.7570 \\
\hline W-C & $<30$ & 185.5578 & 27.3719 & 13.7034 & 10.6546 & 2.8084 & 48.0192 \\
\hline 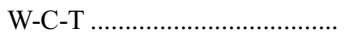 & all & 14.4250 & 2.8392 & 1.8906 & 1.7544 & 1.1566 & 4.4132 \\
\hline 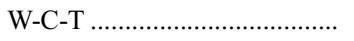 & $<30$ & 27.1182 & 4.6733 & 2.7873 & 2.5053 & 1.2265 & 7.6621 \\
\hline 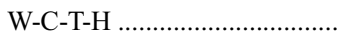 & all & 9.4000 & 5.4792 & 1.6167 & 1.4913 & 1.1282 & 3.8231 \\
\hline 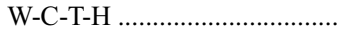 & $<30$ & 13.6420 & 7.8132 & 2.0239 & 1.8904 & 1.1632 & 5.3065 \\
\hline W-C-T-H & $>45$ & 2.0515 & 1.5306 & 1.0962 & 1.1211 & 1.0938 & 1.3787 \\
\hline
\end{tabular}

Notes.-Col. (1): $\mathrm{W}=W M A P$ data, $\mathrm{C}=\mathrm{CMB}$ map defined in eq. (2), $\mathrm{T}=$ Template fits, $\mathrm{H}=$ Haze defined in $\S$ 3.3. Col. (2): Galactic latitude cut, in addition to mask defined in $\S 2$.6. Cols. (3)-(7): $\chi^{2} /$ dof for the $5 W M A P$ bands in $1^{\circ}$ beams. Col. (8): Mean $\chi^{2}$ for the 5 bands. In $\mathrm{W}$ band the $\chi^{2}$ is dominated by imperfect CMB removal. In other bands imperfect foreground removal is dominant, but the model reduces $|b|<30^{\circ}$ excess variance (above measurement noise) in the data by factors of 46, 30, 130, and 60 in $\mathrm{K}-\mathrm{V}$ bands, respectively.

Row (6).--Residual, after subtracting $\mathrm{CMB}, \mathrm{H} \alpha$, soft synchrotron, FDS99 dust, and the putative spinning dust, is nicknamed "the haze." It looks nearly the same in every band and has the spectrum of free-free emission. A template for the haze is produced from the average fit residuals in the $\mathrm{K}$ and $\mathrm{Ka}$ bands, and subtracted from all five WMAP bands with a free-free spectrum. There are no free parameters in this operation, other than one value per sky pixel determined from the $\mathrm{K}$ and Ka maps.

Row (7).- The WMAP residuals after subtracting all above templates show very little remaining other than measurement noise. The one exception is residual emission from $\zeta \mathrm{Oph}$, which appears to peak at $41 \mathrm{GHz}$ and roll off to either side (relative to free-free). The point source mask used in the analysis is shown in the lower right-hand panel.

\section{INTERPRETATION}

The three important results of this analysis are that (1) the quality of the fit is very high, (2) the dust-correlated component has a reasonable spectrum to be spinning dust, and (3) a "haze" unassociated with $\mathrm{H} \alpha$ appears to be present in the data.

\subsection{Quality of Fit}

The quality of the fit is remarkable. All that is subtracted from each sky map is a CMB template; a map with free-free spectrum (the $\mathrm{H} \alpha$ based template plus the haze); soft synchrotron, which is hardly controversial at low frequencies and unimportant at high frequencies; FDS99 dust emission extrapolated from the FIRAS fit; and emission strictly correlated with FDS99 dust times $T_{\text {dust }}^{2}$. This last component is the only one that can contain hard synchrotron emission or spinning dust emission-the others are all well constrained and physically understood. The surprisingly small residuals imply that the physical mechanism primarily responsible for this emission is closely tied to the dust grains themselves (e.g., spinning dust) rather than a mechanism relying on the spatial correlation of dust and some other emission source (e.g., hard synchrotron emission).

In doing this fit, we have not allowed the spectral dependence of any component to float from pixel to pixel, as the WMAP team MEM analysis did (Bennett et al. 2003). Although it may seem overly conservative to fix the spectral dependence of each component, this rigidity in the fit allowed the discovery of the free-free haze, and the quality of the resulting fit indicates that spectral variation of the components is not required to fit $W M A P$ data off the plane. Because the templates were applied directly rather than as priors to a MEM analysis, evaluation of $\chi^{2}$ is straightforward. Values given in Table 1 are for $1^{\circ}$ FWHM Gaussian beams; this reduces measurement noise to approximately $15 \mu \mathrm{K}$ per beam in each band. Because of the CMB and haze subtraction, the residual in each band is actually a superposition of the foreground templates and five $W M A P$ bands appropriately weighted. The smoothed WMAP noise for each band is then combined in a weighted quadrature sum to produce the expected noise in the five residual maps. This process assumes that the five $W M A P$ bands have uncorrelated noise and that the $\mathrm{H} \alpha$, Haslam et al. (1982), and FDS99 templates have no measurement noise. The fact that $\chi^{2}$ per degree of freedom (dof) is of order unity at high latitude (Table 1, last row) indicates that these assumptions are justified in the $\mathrm{Q}, \mathrm{V}$, and $\mathrm{W}$ bands, and less so in the $\mathrm{K}$ and $\mathrm{Ka}$ bands. At $|b|<30^{\circ}$ the $\chi^{2} /$ dof is formally poor (13.6 at $K, 7.8$ at $\mathrm{Ka}$, and 2 at $\mathrm{Q})$ yet represents removal of $98 \%, 97 \%$, and $99 \%$ of the variance in the $W M A P$ data, respectively. It is significant that these values are with respect to the $1^{\circ} \mathrm{FWHM}$ smoothed data; the residual variance of $60 \mu \mathrm{K}$ per beam at the $\mathrm{K}$ band is less than half of the median measurement noise per pixel and relatively smaller in the other bands.

The residuals in the Bennett et al. (2003) analysis are also impressively small. It is impossible to compare the results of this study to the Bennett et al. MEM analysis, which fitted several numbers for each pixel on the sky (constrained by prior templates). Such a MEM analysis does not allow for an easy interpretation of $\chi^{2}$, so a smaller fit residual would not imply the superiority of one foreground parameterization over the other. In fact, the same freedom that allows the MEM to minimize foreground contamination of the cosmological signal may actually inhibit the physical interpretation of the foreground signal by allowing an overly simple model to fit the data.

\subsection{Spinning Dust Spectrum}

Draine \& Lazarian $(1998,1999)$ chose to phrase the comparison between theory and observation in terms of emissivity per $\mathrm{H}$ atom, using units of $\mathrm{Jy} \mathrm{cm}^{2} \mathrm{sr}^{-1}$ per $\mathrm{H}$ in their work. ${ }^{7}$ This ratio is appealing to theorists because it directly relates

\footnotetext{
${ }^{7}$ Plots in the early Draine \& Lazarian articles are incorrectly labeled "Jy $\mathrm{sr}^{-1}$ per H," an error repeated by Finkbeiner et al. (2002).
} 
TABLE 2

Correlation Slopes

\begin{tabular}{cccc}
\hline \hline $\begin{array}{c}\text { Band } \\
(1)\end{array}$ & $\begin{array}{c}\text { Frequency } \\
(\mathrm{GHz}) \\
(2)\end{array}$ & $\begin{array}{c}\text { Spinning Dust } \\
\left(\mathrm{MJy} \mathrm{s}^{-1} \tau_{100 \mu}^{-1}\right)\end{array}$ & $\begin{array}{c}\text { Spin + Thermal } \\
(3)\end{array}$ \\
\hline $\left.\mathrm{MJy} \mathrm{sr} \tau_{100 \mu}^{-1}\right)$ \\
$(4)$
\end{tabular}

Notes. - Col. (1): WMAP band name. Col. (2): Effective central frequency for free-free spectrum. Col. (3): Cross-correlation slope of spinning dust component per $100 \mu \mathrm{m}$ optical depth. Col. (4): Sum of spinning dust and thermal (vibrational) dust predicted by Finkbeiner et al. (1999) per $\tau_{100 \mu}$. These data are plotted in Fig. 2.

the dust model to chemical abundances in the ISM. However, measuring the hydrogen column density, including molecular, atomic, and ionized forms, is technically difficult. Finkbeiner et al. (2002) measured cross-correlation slopes of emission against the SFD98 dust map, and converted to $N(\mathrm{H})$ using $1 \mathrm{mag} E(B-V)=8 \times 10^{21} N(\mathrm{H})$. Although this conversion is appropriate for diffuse high-latitude material, the gas-to-dust ratio is known to vary in general, and phrasing measurements

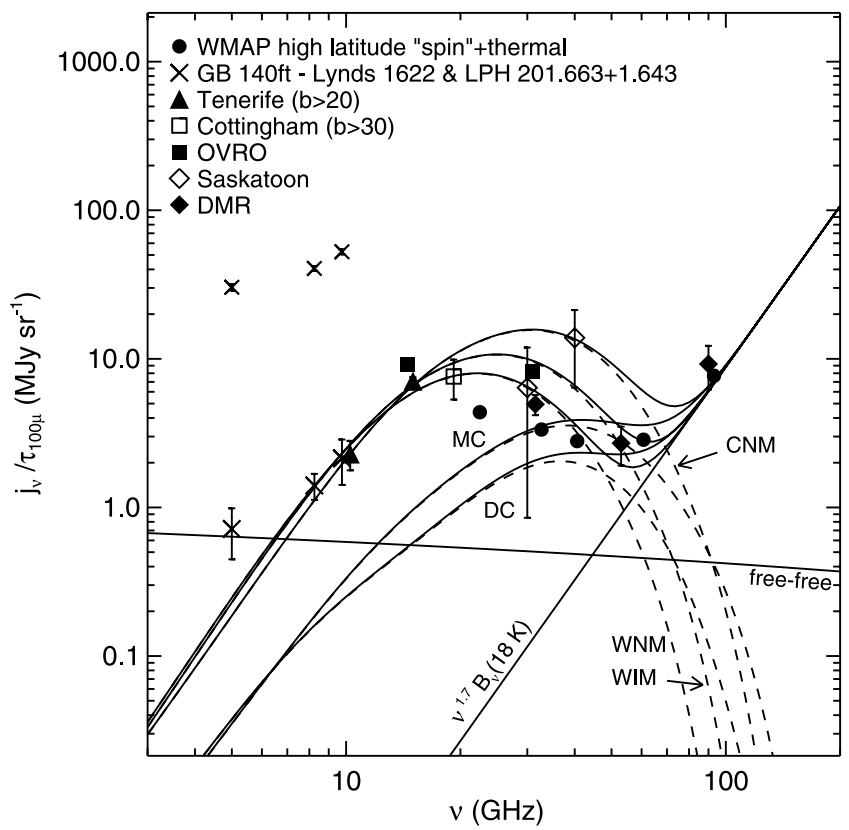

FIG. 2.-Model dust emissivity per $\tau_{100 \mu}$ for DC, MC, CNM, WNM, and WIM conditions (as in Draine \& Lazarian 1998, Fig. 9) with (solid lines) and without (dashed lines) contribution from vibrational dust at mean temperature. For comparison with the Draine \& Lazarian models, unit $\tau_{100 \mu}$ corresponds to $N(\mathrm{H})=2.13 \times 10^{24} \mathrm{~cm}^{-2}$. Gray line is emission from free-free for $\left\langle n_{e} n_{p}\right\rangle /\left\langle n_{\mathrm{H}}\right\rangle=$ $0.01 \mathrm{~cm}^{-3}$ averaged along the line of sight. WMAP data from Table 2 (including thermal dust) are overplotted ( filled circles). Also shown are measurements from the COBE/DMR ( filled diamonds) from Finkbeiner et al. (1999), similar to Kogut et al. (1996); Saskatoon (open diamonds; de Oliveira-Costa et al. 1997); the Cottingham \& Boughn $19.2 \mathrm{GHz}$ survey (open square; de Oliveira-Costa et al. 1998); OVRO data ( filled squares; Leitch et al. 1997); Tenerife data (triangles; de Oliveira-Costa et al. 1999); GB 140 foot (43 m) (crosses; Finkbeiner et al. 2002). The OVRO points have been lowered a factor of 3 relative to Draine \& Lazarian (1998, Fig. 9), because the unusual dust temperature near the north celestial pole caused an underestimate of $\tau_{100 \mu}$ along those lines of sight. Given the large range of model curves, all measurements are consistent with some superposition of spinning dust, vibrational dust, and free-free emission. [See the electronic edition of the Journal for a color version of this figure.]

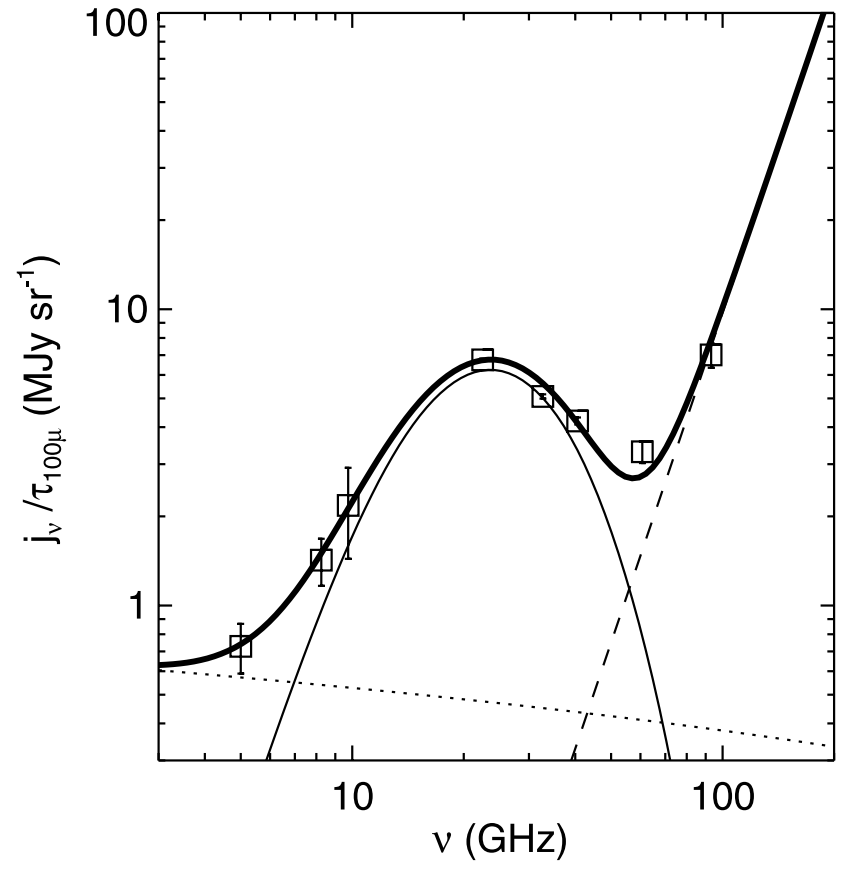

FIG. 3.-LDN 1622. Green Bank $(5,8,10 \mathrm{GHz})$ measurements in a $6^{\prime}$ beam (Finkbeiner et al. 2002) and WMAP (23-94 GHz) data points smoothed to a $60^{\prime}$ beam (symbols), each with statistical $1 \sigma$ error bars only. Thermal dust estimate from Finkbeiner et al. (1999) (dashed line), free-free estimate (dotted line), spinning dust (lower solid line), and sum of free-free, thermal dust, and spinning dust (upper solid line). The spinning dust model is a superposition of two Draine \& Lazarian models: $0.6 \times \mathrm{WNM}$ plus $0.11 \times \mathrm{CNM}$ model.

in terms of $E(B-V)$ or $N(\mathrm{H})$ when they have nothing to do with either has led to confusion.

In this work, we stay as close as possible to the empirical data and state emissivity per dust in terms of optical depth at $100 \mu \mathrm{m}$, or $\tau_{100 \mu}$. This optical depth is defined with respect to a blackbody at the reference dust temperature $T_{\text {ref }}=18.175 \mathrm{~K}$ used in SFD98. This temperature is the median high-latitude dust color temperature determined from the DIRBE $100 \mu \mathrm{m} / 240 \mu \mathrm{m}$ intensity ratio assuming a $\nu^{2}$ emissivity law. For the SFD 98 calibrations, a $\tau_{100 \mu}$ of unity corresponds to $266 \mathrm{mag} E(B-V)$ or $2.13 \times 10^{24} N(\mathrm{H})$, so the reader can convert Draine \& Lazarian models and previously published measurements to $j_{\nu} / \tau_{100 \mu}$ in Jy sr ${ }^{-1}$ by multiplying by $2.13 \times 10^{24}$.

The cross-correlation coefficients for the $\operatorname{FDS} 99 \times T_{\text {dust }}^{2}$ spinning dust template are given in Table 2 . These can be added to the FDS99 thermal dust prediction, where the sum is taken at dust temperature $T_{\text {ref }}=18.175 \mathrm{~K}$. In Figure 2 the dustcorrelated emission per $\tau_{100 \mu}$ is shown for five Draine \& Lazarian (1998) models of electric dipole emission from spinning dust, along with the cross-correlation points from $W M A P$ and other projects. Note that the thermal dust emission and "spinning dust" emission have been combined for WMAP, just as they have been for other experiments (intentionally or not). The $W M A P$ data points fall about a factor of 2 lower than the warm neutral medium (WNM) model. These WMAP points represent the average high-latitude sky, so there is no reason to expect them to agree with any particular Draine \& Lazarian model, but rather a superposition.

Two spinning dust candidates previously studied with the Green Bank 140 foot (43 m) telescope (Finkbeiner et al. 2002) are also shown: LDN 1622 (Fig. 3, see also Hildebrand \& Kirby 2003) and [LPH96] 201.663+1.643 from the Lockman et al. (1996) catalog of diffuse $\mathrm{H}$ il regions (Fig. 4). A superposition 


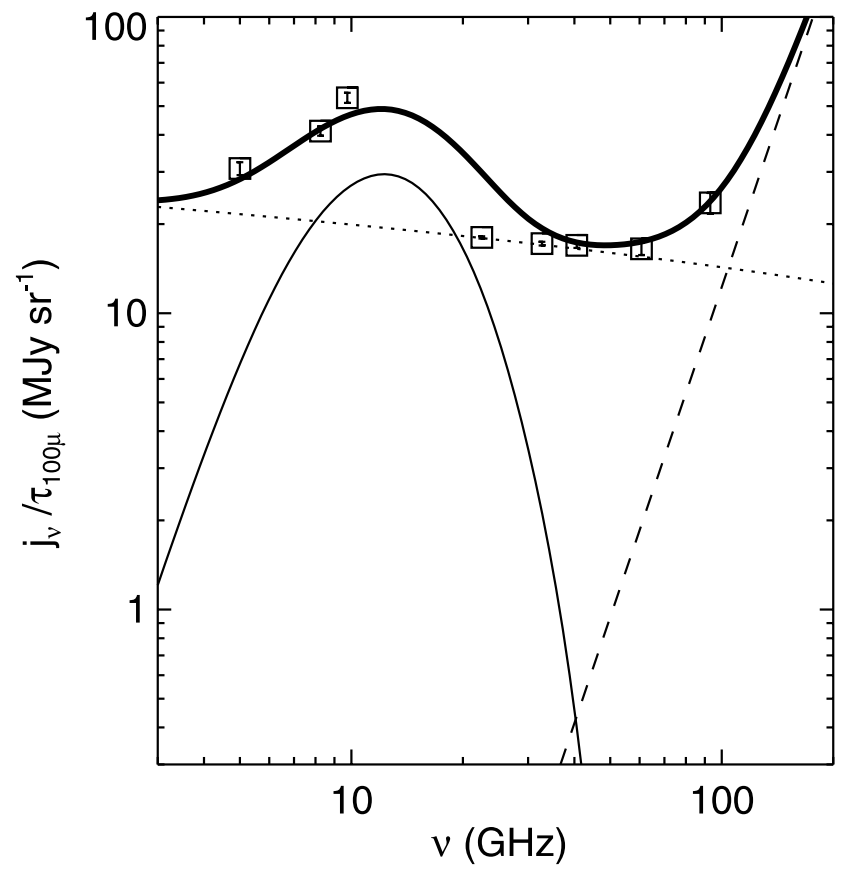

FIG. 4.-[LPH96] 201.663+1.643. Green Bank $(5,8,10 \mathrm{GHz})$ and $W M A P$ (23-94 GHz) data points (symbols), thermal dust estimate from Finkbeiner et al. (1999) (dashed line), free-free estimate (dotted line), spinning dust (lower solid line), and sum of free-free, thermal dust, and spinning dust (upper solid line). None of the published spinning dust models come close to fitting this object. For reference, the CNM model is shown, shifted in frequency and amplitude. This curve may not represent a physical spinning model for any reasonable choice of parameters. Green Bank points are $6^{\prime}$ and $W M A P$ is smoothed to $60^{\prime}$, so deviation of the fit from $W M A P$ points may be due to beam mismatch between Green Bank and $W M A P$, but the outlier at $10 \mathrm{GHz}$ is significant. of the WNM and CNM models plus a small amount of free-free yields a reasonable fit for LDN 1622. Note that the thermal dust level is not being fitted, merely extrapolated from the far-IR according to the FDS99 FIRAS fit. Spinning dust models have a few free parameters to tune, and some models may come closer to fitting the LPH96 cloud, although this will require further theoretical work. Lacking a physical model for this object, a curve (the Draine \& Lazarian WNM model shifted down a factor of 1.8 in frequency and up a factor of 3.7 in amplitude) is shown in Figure 4 for comparison. Neither of these objects would be well fit by a superposition of synchrotron, free-free, and thermal dust without including spinning dust.

\subsection{The Haze}

A rather substantial amount of emission (both positive and negative) was attributed above to the "haze," without any interpretation. As the spectrum of this component most resembles free-free (Fig. 5), the straightforward interpretation is that it constitutes the error in the $\mathrm{H} \alpha$ based free-free template. Because the spatial template is orthogonal to the others by construction, it contains positive and negative parts. Some error is to be expected both from variation in warm gas temperature about the mean $T_{\text {gas }}=5500 \mathrm{~K}$ used and from the fact that some gas is hot enough $\left(>10^{6} \mathrm{~K}\right)$ that the $\mathrm{H} \alpha$ recombination line is strongly suppressed. This need for hot gas applies especially to the region south of the Galactic center, where there is significant emission seen in the haze, but none of the other templates have a similarly extended feature of significant brightness.

Further study of this diffuse haze, perhaps using $\mathrm{O}$ vi absorption measurements toward distant stars below the Galactic center, may be able to verify this hypothesis about its origin. We emphasize that the tentative identification of the haze as free-free is based only on its spectral shape, and if WMAP polarization data show a significant polarization, that would suggest very hard synchrotron $\left(\sim \nu^{-0.1}\right)$ emission as a source. Further consideration of the haze is deferred to another paper.
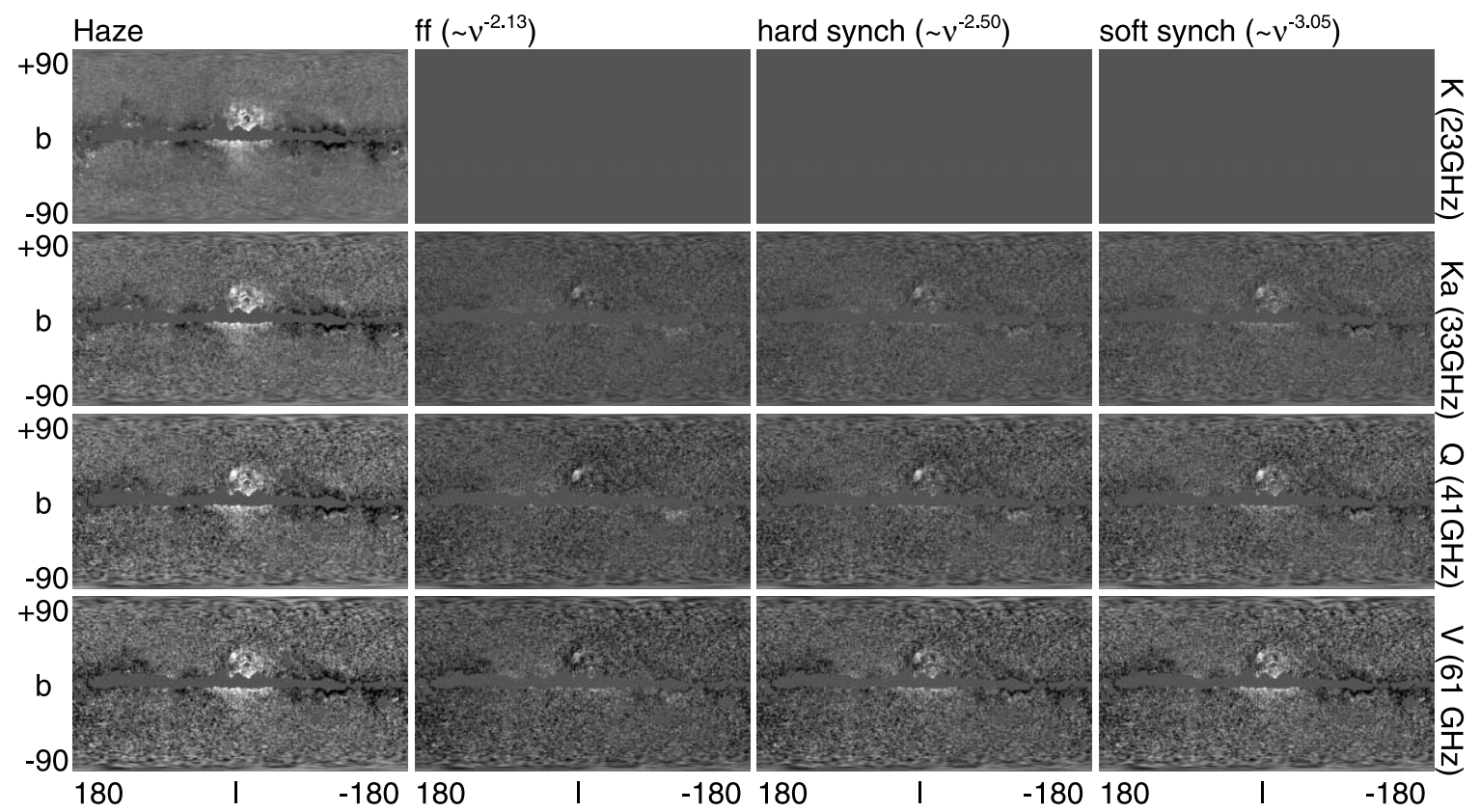

Fig. 5.-Haze is determined in $4 W M A P$ bands by subtracting CMB, soft synchrotron (Haslam et al. [1982] template), free-free (H $\alpha$ template), and spinning dust. Using the K-band haze as a template, it is then subtracted from Ka, Q, and V bands assuming various power laws. A free-free spectrum fits most of the sky well, apart from the $\zeta$ Oph cloud $(l, b)=\left(5^{\circ}, 25^{\circ}\right)$. See $\S 3.3$. 
A HEALPix map of the K-band haze template used in Figure 1 , defined as $\left(T_{K}+2.186 T_{\mathrm{Ka}}\right) / 2$, is available on the Web. ${ }^{8}$

\section{CONCLUSIONS}

We have performed an analysis of the WMAP temperature anisotropy data, using free-free, soft synchrotron, FDS99 thermal dust, and spinning dust as templates. We find the following:

1. The FDS99 thermal (nonmagnetic) dust prediction is good on average over the high-latitude sky at $94 \mathrm{GHz}$ and even in dark cloud LDN 1622 and H II region [LPH96] 201.663+1.643.

2. Free-free is fit by $T=5500 \mathrm{~K}$ ionized gas (rather cool, but see, e.g., Heiles et al. 2000) plus a haze toward the Galactic center with a free-free spectrum. This is the first detection of emission from this component. Further study is needed to test whether this component is indeed free-free or perhaps a very hard synchrotron component.

3. The residual dust-correlated emission is well fit by $T_{\text {dust }}^{3}$ times the dust column density over most of the sky. Such a model, along with free-free, soft synchrotron, and FDS99 thermal dust, accounts for $\sim 98 \%$ of the variance in the WMAP data.

4. There is no conflict between the general idea of spinning dust emission and the $W M A P$ data. In spite of the Bennett et al. (2003) statement that the Draine \& Lazarian CNM model accounts for less than $5 \%$ of the ISM emission (which is true), spinning dust remains a reasonable hypothesis both morphologically and spectrally.

5. The Green Bank Galactic Plane Survey GPA data at 8 and $14 \mathrm{GHz}$ (Langston et al. 2000) rule out hard synchrotron as a majority component in the diffuse ISM in the WMAP bands (see companion paper, Finkbeiner et al. 2004). This leaves spinning dust as a viable alternative.

\footnotetext{
${ }^{8}$ See http://skymaps.info.
}

The WMAP data alone cannot establish the relative levels of spinning dust, magnetic dust, and hard synchrotron emission, since any of these theories can be adjusted to explain the data. However, objects such as LDN 1622 (Fig. 3) are clearly not dominated by synchrotron in the WMAP bands and are well fitted by a superposition of spinning dust models. Therefore, the dominance of spinning dust at $23 \mathrm{GHz}$ is a good working hypothesis. Further data (including surveys at lower frequencies, better WMAP signal to noise, and WMAP polarization data coming soon) will help lift the remaining degeneracies and determine the identity of the dust-correlated microwave emission from "foreground(s) X."

I am indebted to Bruce Draine, Ed Jenkins, and David Schlegel for encouragement and helpful advice. Roger Hildebrand called the WMAP spectrum of LDN 1622 to my attention, and provided other insights. Lyman Page, Chuck Bennett, and Gary Hinshaw provided helpful comments on the proper use of the WMAP data in this work. Conversations with Angelica de Oliveira-Costa and Max Tegmark were illuminating. Comments from an anonymous referee substantially improved the text. Some of the results in this paper were derived using HEALPix (Górski et al. 1999). This research made use of the NASA Astrophysics Data System (ADS) and the IDL Astronomy User's Library at Goddard. ${ }^{9}$ D.P.F. is a Hubble Fellow supported by HST-HF-00129.01-A during much of this project. He is currently supported by NASA LTSA grant NAG5-12972, the Russell Fellowship, and the Cotsen Fellowship of the Society of Fellows in the Liberal Arts at Princeton University.

\footnotetext{
${ }^{9}$ See http://idlastro.gsfc.nasa.gov/.
}

\section{REFERENCES}

Agladze, N. I., Sievers, A. J., Jones, S. A., Burlitch, J. M., \& Beckwith, S. V. W. 1996, ApJ, 462, 1026

Banday, A. J., Dickinson, C., Davies, R. D., Davis, R. J., \& Górski, K. M. 2003, MNRAS, 345, 897

Bennett, C. L., et al. 2003, ApJS, 148, 97

Brodd, S., Fixsen, D. J., Jensen, K. A., Mather, J. C., \& Shafer, R. A., ed. 1997, $C O B E /$ FIRAS Explanatory Supplement (COBE Ref. Pub. 97-C; Greenbelt: NASA/GSFC)

de Oliveira-Costa, A., Kogut, A., Devlin, M. J., Netterfield, C. B., Page, L. A., \& Wollack, E. J. 1997, ApJ, 482, L17

de Oliveira-Costa, A., Tegmark, M., Page, L., \& Boughn, S. 1998, ApJ, 509, L9

de Oliveira-Costa, A., et al. 1999, ApJ, 527, L9

$$
\text { 2002, ApJ, 567, } 363
$$

$$
\text { 2004, ApJ, 606, L89 }
$$

Dennison, B., Simonetti, J. H., \& Topasna, G. 1998, Publ. Astron. Soc. Australia, 15,147

Draine B. T., \& Lazarian, A. 1998, ApJ, 508, 157 1999, ApJ, 512, 740

Draine, B. T., \& Lee, H. M. 1984, ApJ, 285, 89

Erickson, W. C. 1957, ApJ, 126, 480

Ferrara, A., \& Dettmar, R.-J. 1994, ApJ, 427, 155

Finkbeiner, D. P. 2003, ApJS, 146, 407

Finkbeiner, D. P., Davis, M., \& Schlegel, D. J. 1999, ApJ, 524, 867 (FDS99)

Finkbeiner, D. P., Langston, G. I., \& Minter A. H. 2004, ApJ, in press (astro$\mathrm{ph} / 0408292$ )

Finkbeiner, D. P., Schlegel, D. J., Frank, C., \& Heiles, C. 2002, ApJ, 566, 898

Fixsen, D. J., Cheng, E. S., Gales, J. M., Mather, J. C., Shafer, R. A., \& Wright, E. L. 1996, ApJ, 473, 576

Gaustad, J. E., McCullough, P. R., Rosing, W., \& Van Buren, D. 2001, PASP, 113,1326
Górski, K. M, Hivon, E., \& Wandelt, B. D. 1999, in MPA/ESO Cosmology Conf., Evolution of Large-Scale Structure, ed. A. J. Banday, R. K. Sheth, \& L. N. da Costa (Garching: ESO), 37

Haffner, L. M., Reynolds, R. J., Tufte, S. L., Madsen, G. J., Jaehnig, K. P., \& Percival, J. W. 2003, ApJS, 149, 405

Haslam, C. G. T., Stoffel, H., Salter, C. J., \& Wilson, W. E. 1982, A\&AS, 47, 1

Heiles, C., Haffner, L. M., Reynolds, R. J., \& Tufte, S. L. 2000, ApJ, 536, 335

Hildebrand, R., \& Kirby, L. 2003 in ASP Conf. Ser. 309, Astrophysics of Dust, ed. A. N. Witt, G. C. Clayton, \& B. T. Draine (San Francisco: ASP), 515

Hinshaw, G., et al. 2003, ApJS, 148, 135

Jarosik, N., et al. 2003, ApJS, 148, 29

Kogut, A., et al. 1996, ApJ, 464, L5

Kurtz, S., Churchwell, E., \& Wood, D. O. S. 1994, ApJS, 91, 659

Lagache, G. 2003, A\&A, 405, 813

Langston, G., Minter, A., D’Addario, L., Eberhardt, K., Koski, K., \& Zuber, J. 2000, AJ, 119, 2801

Lazarian, A., \& Draine, B. T. 2000, ApJ, 536, L15

Leitch, E. M., Readhead, A. C. S., Pearson, T. J., \& Myers, S. T. 1997, ApJ, 486, L23

Lockman, F. J., Pisano, D. J., \& Howard, G. J. 1996, ApJ, 472, 173 (LPH96)

Masi, S., et al. 2001, ApJ, 553, L93

Press, W. H., Teukolsky, S. A., Vetterling, W. T., \& Flannery, B. P. 1992, Numerical Recipes in C (2nd ed.; Cambridge, UK: Cambridge Univ. Press) Reach, W. T., et al. 1995, ApJ, 451, 188

Schlegel, D. J., Finkbeiner, D. P., \& Davis, M. 1998, ApJ, 500, 525 (SFD98) Spergel, D. N., et al. 2003, ApJS, 148, 175

Spitzer, L. 1978, Physical Processes in the Interstellar Medium (New York: Wiley)

Valls-Gabaud, D. 1998, Publ. Astron. Soc. Australia, 15, 111 